\title{
On Ripple Reduction of PWM Coupled Inductors
}

\author{
Minjie Chen, Senior Member, IEEE
}

\begin{abstract}
This letter investigates the ripple reduction mechanisms of pulsed-width-modulated (PWM) coupled inductors based on the principles of multiphase interleaving. By adopting circuit duality and superposition analysis, the ripple reduction ratio of PWM coupled inductors is, for the first time, presented as a function of the ripple reduction ratio of multiphase interleaving and the coupling coefficient of the coupled inductor. The analysis simplifies the assumptions used in previous study, and results in concise and intuitive description of the ripple reduction mechanisms of coupled inductors. The results provide useful guidelines for designing interleaved and coupled PWM converters.
\end{abstract}

Index Terms - circuit interleaving, magnetic coupling, duality

\section{INTRODUCTION}

$\mathbf{I}$ NTERLEAVING can improve the performance of paralleled power converters. In an interleaved multicell system [1]-[4], the cells are operated at the same switching frequency with their switching waveforms displaced in phase over a switching period. For most pulse-width-modulated (PWM) topologies, interleaved operation of $M$ cells yields an $M$ fold increase in fundamental current ripple frequency, and a reduction in peak ripple magnitude by a factor $M$ of or more compared to synchronous operation. Although interleaving allows much smaller overall inductor size and faster response at the input and the output, the component behavior and performance within each individual cell is the same as in a single buck converter. Coupling the inductors between multiple interleaved PWM cells can extend the ripple reduction achieved in the input or output to ripples within each individual cells [5]-[7]. It has been shown that strong coupling between multiple inductors can greatly reduce the inductor size, reduce the loss, and improve the control bandwidth of a variety of multicell PWM converters.

Models for multiphase PWM coupled inductors are usually complicated. This is in part because of the intrinsic sophisticated magnetic coupling relations, and in part because of the way how these relations are approached and presented. [8] compares and unifies a variety of different methodologies for analyzing multiphase coupled inductors for different purposes. However, the derivation in [8] require a few different types of assumptions. The current ripple reduction mechanisms of magnetic coupling is still sophisticated and non-intuitive.

This letter aims at demystifying the ripple reduction mechanisms of PWM coupling. By adopting circuit duality and superposition analysis, the ripple reduction ratio of PWM magnetic coupling is shown as a function of the ripple reduction ratio of multiphase interleaving and the coupling coefficient. The concise design equations provide useful guidelines for designing interleaved and coupled PWM converters.

M. Chen is with the Department of Electrical and Computer Engineering and Andlinger Center for Energy and the Environment at Princeton University, Princeton, NJ 08540, USA.

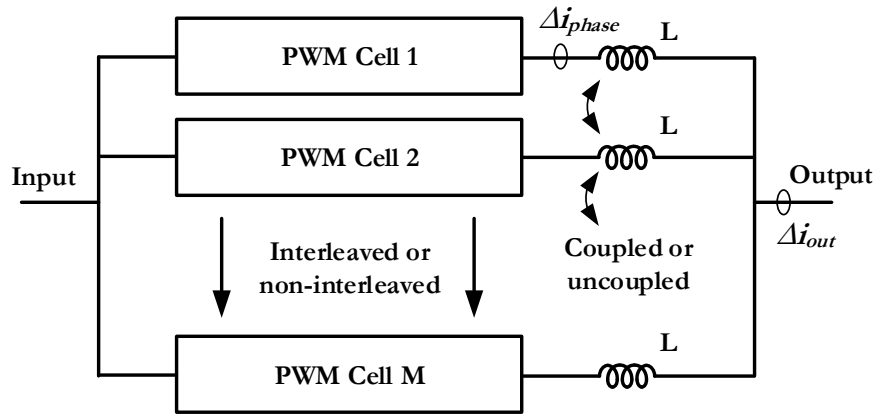

Fig. 1. A interleaved or non-interleaved, coupled or uncoupled PWM converter. The phase and output current ripple are the focuses of this paper.

\section{RipPle REDUCTION OF PARALLEL INTERLEAVING}

Fig. 9 shows the architecture of a multiphase PWM converter with coupled/uncoupled inductors and interleaved/noninterleaved operations. As shown in [1]-[4], the total output current ripple amplitude after ripple cancellation is reduced compared to the ripple in a standalone converter by a factor that depends on the relationship between the duty ratio $D$ and the number of phases $M$. Define an integer index such that $\frac{k}{M} \leq D \leq \frac{k+1}{M}$, the output ripple of an interleaved uncoupled multiphase buck converter $\left(\Delta i_{\text {out }}^{\text {interleaved }}\right)$ is reduced relative to that of non-interleaved uncoupled converter $\left(\Delta i_{\text {out }}^{\text {non-interleaved }}\right)$ with the same phase inductance by a factor $\Gamma$, as a function of $k$, duty ratio $D$, and number of phases $M$ :

$$
\Gamma \stackrel{\text { def }}{=} \frac{\Delta i_{\text {out }}^{\text {interleaved }}}{\Delta i_{\text {out }}^{\text {non-interleaved }}}=\frac{(k+1-D M)(D M-k)}{(1-D) D M^{2}} .
$$

$\Gamma$ quantifies the benefits of interleaving for output current ripple reduction. Fig. 2 plots $\Gamma$ as a function of $M$ and $D$. $\Gamma$ decreases as $M$ increases. When the duty cycle is near an integer multiple of $1 / M, \Gamma$ approaches zero, indicating fully eliminated output current ripple.

\section{Ripple REDUCtion OF PWM COUPLEd INDUCTORS}

The benefits of ripple reduction in multiphase coupling have been studied in [8]. Here we derive the benefits of ripple ratio reduction by adopting circuit duality and superposition. Compared to the derivation provided in [8], this derivation relies on fewer assumption and offers better design insights. Take the coupled inductor in Fig. 3 as an example. If the core has sufficiently high permeability, or if the top and and bottom bars of the core have sufficiently large cross sectional area, we can neglect the reluctance of those parts of the core, resulting in a simplified reluctance model. In this model, each winding leg has a reluctance $\mathcal{R}_{L}$. All leakage reluctance are represented by $\mathcal{R}_{C}$ This simplified model captures the 


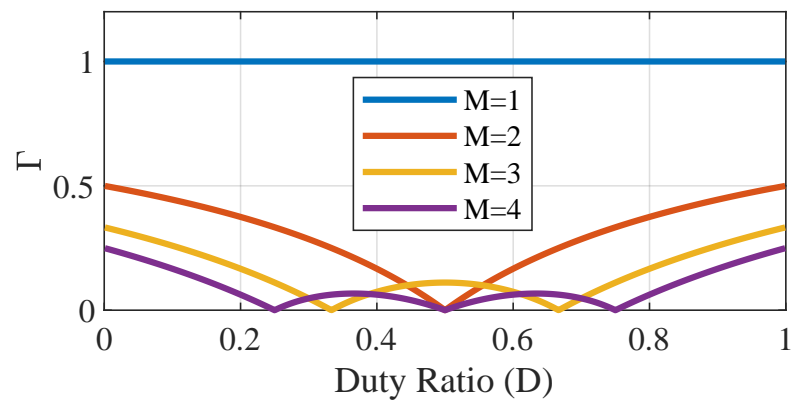

Fig. 2. Output current ripple reduction factor $(\Gamma)$ for an $M$-phase interleaved buck converter with duty ratio $D$. $\Gamma$ quantifies the benefit of interleaving for output current ripple reduction in multiphase buck converters.
Multiphase Coupled Inductor

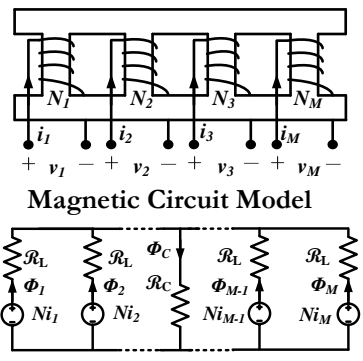

Inductance Dual Model $i_{C} L_{\mathrm{C}}=1 / \mathcal{R}_{\mathrm{C}}$

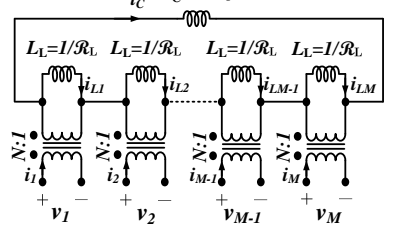

Permeance-Capacitor Model

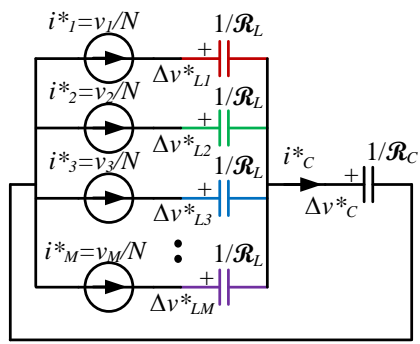

Interleaved Excitations

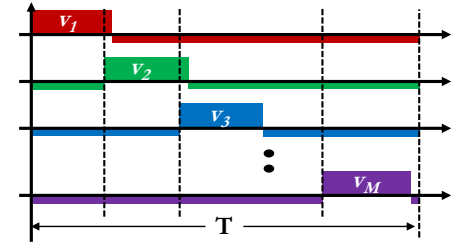

Fig. 3. A multiphase coupled inductor with its magnetic circuit model, inductance dual model, and permeance-capacitor model.

main features of the circuit behavior of multiphase coupled inductors. The topology of the electrical circuit model arising directly from the magnetic circuit model is the topological dual, with reluctances replaced by inductors. This model is attractive not only for its simplicity, but also because each inductor in the model corresponds to a specific physical part of the structure. The magnetic flux in each inductor is linearly related to the magnetic flux in the corresponding part of the core, and this facilitates modeling of non-ideal magnetic behavior including core losses and saturation.

Apply duality to inductance dual model again result in a capacitor network driven by gyrators, which is sometimes referred to as a permeance-capacitor model with capacitance values equal to the magnetic permeance [10]. Current $i^{*}$ in this model represents voltage $v$ in the circuit domain; voltage $v^{*}$ in this model represents current $i$ in the circuit domain. We apply superposition analysis to this model to analyze the ripple reduction mechanisms and their benefits.

\section{A. Phase Current Ripple Reduction}

Figure 4 illustrates the key steps of the superposition analysis. Assume $v_{1}, v_{2}, \ldots, v_{M}$ are identical PWM square wave

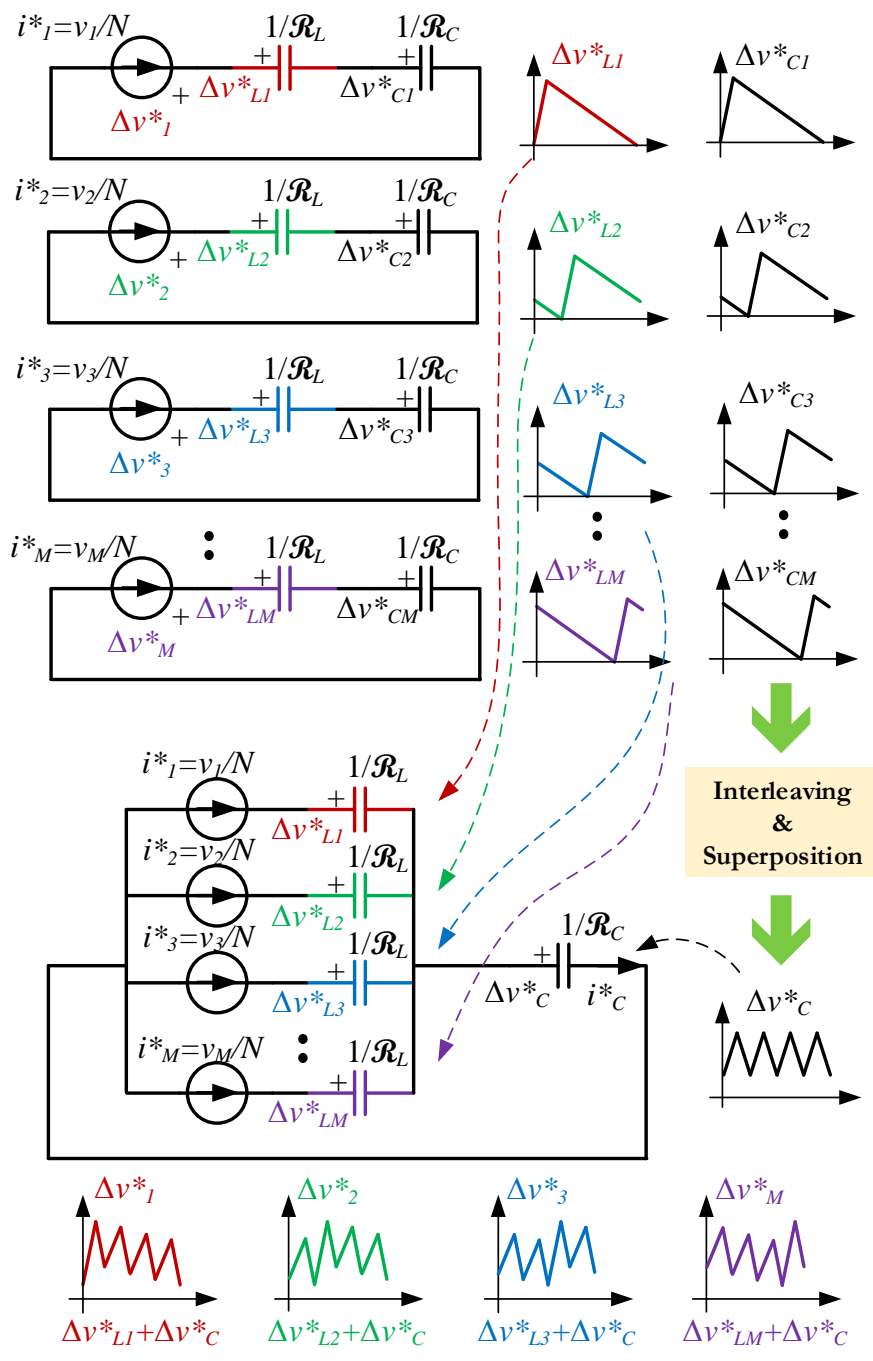

Fig. 4. Superposition analysis for the permeance-capacitor model of a $M$ phase coupled inductor with interleaved operation.

voltage with their integrated volt-second within one switching cycle as $\sigma$, and each winding has a single turn $N=1$. $M$ superposition subcircuits are created. In each subcircuit, the sqaure wave current results in a triangular voltage ripple on the two series-connected capacitors. The voltage ripples on the two capacitors in each subcircuit are

$$
\begin{aligned}
\Delta v_{L x}^{*} & =\sigma \mathcal{R}_{L}, \\
\Delta v_{C x}^{*} & =\sigma \mathcal{R}_{C} .
\end{aligned}
$$

Each of the branch capacitor (the $1 / \mathcal{R}_{L x}$ elements) is only excited by the current source that it is connected in series with, and the shared capacitor is excited by all current sources. Its overall voltage is the summation of its voltage waveform in all subcircuits. If the $M$ phases are not interleaved, the overall voltage ripple $\Delta v_{C}^{*}$ is $M \sigma \mathcal{R}_{C}$. If the $M$ phases are interleaved [4], the overall voltage ripple reduces to

$$
\Delta v_{C}^{*}=\Gamma M \sigma \mathcal{R}_{C} .
$$

Since the ripple of $v_{C}^{*}$ and $v_{L x}^{*}$ are synchronized to the PWM switching clock, the peak-to-peak ripple of the voltage 


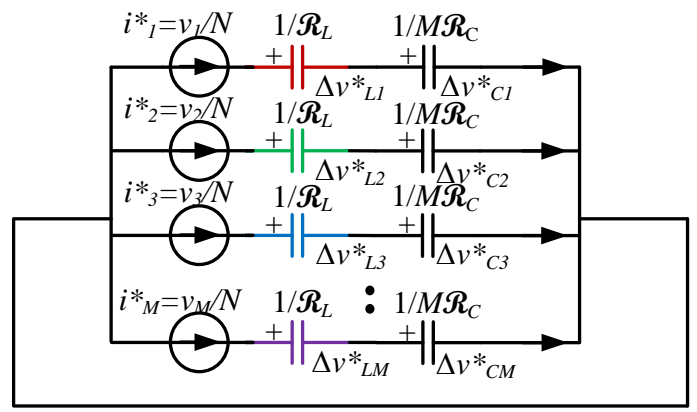

Fig. 5. Models for the circuit if all phases are non-interleaved.

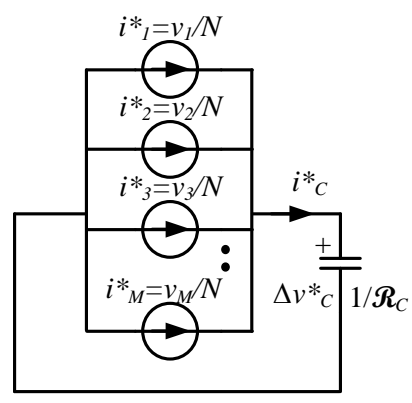

Strongly Coupled, $\beta \rightarrow+\infty$

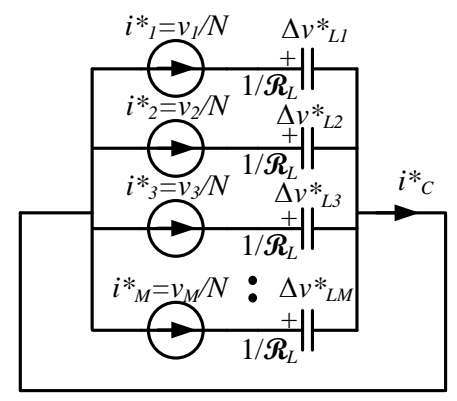

Weakly Coupled, $\beta \rightarrow 0$
Fig. 6. Simplified circuit model of Fig. 5 when $\beta \rightarrow+\infty$ and $\beta \rightarrow 0$.

across the current source, which equals the phase current ripple in each winding, is the summation of $\Delta v_{C}^{*}$ and $\Delta v_{L x}^{*}$

$$
\Delta i_{\text {phase }}^{\text {interleaved }}=\sigma\left(\Gamma M \mathcal{R}_{C}+\mathcal{R}_{L}\right) .
$$

If the $M$ phases are not interleaved, the permeance-capacitor model can be analyzed as shown in Fig. 5 with $M$ synchronized identical phases. The phase current ripple becomes:

$$
\Delta i_{\text {phase }}^{\text {non-interleaved }}=\sigma\left(M \mathcal{R}_{C}+\mathcal{R}_{L}\right) .
$$

Define $\beta=M \mathcal{R}_{C} / \mathcal{R}_{L}$, the ratio between the phase current ripple of a $M$ phase coupled inductor with interleaved or noninterleaved operation is

$$
\gamma_{\text {phase }} \stackrel{\text { def }}{=} \frac{\Delta i_{\text {phase }}^{\text {interleaved }}}{\Delta i_{\text {phase }}^{\text {non-interleaved }}}=\frac{\Gamma M \mathcal{R}_{C}+\mathcal{R}_{L}}{M \mathcal{R}_{C}+\mathcal{R}_{L}}=\frac{1+\beta \Gamma}{1+\beta} .
$$

This definition of $\gamma_{\text {phase }}$ does not rely on the assumption on transient performance as described in [8], which compares coupled and non-coupled inductors by assuming identical transient performance for the two PWM converters. (7) indicates that the phase current ripple reduction in PWM coupled inductors has a strong relationship with interleaving. The voltage ripple of the shared capacitor in the summed subcircuit adds together similar to adding interleaved current with their ripples cancelled by a factor of $\Gamma$. The reduced voltage ripple in the $\mathcal{R}_{C}$ element reduces the voltage ripple across the gyrators in the permeance-capacitor model, yieldly reduced current ripple in the circuit domain. Based on (7), if the coupling is strong, i.e., $\beta \rightarrow+\infty$, and $\gamma_{\text {phase }}$ approaches $\Gamma$. This can be illustrated by omitting the branch capacitors in Fig. 5, as shown in Fig. 6. In the magnetic domain, the $d \Phi / d t$
TABLE I

NORMALIZED OUTPUT CURRENT RIPPLE FOR UNCOUPLED AND COUPLED, NON-INTERLEAVED AND INTERLEAVED PWM CONVERTERS

\begin{tabular}{c|cc|cc}
\hline Normalized & \multicolumn{2}{|c|}{ Output Current } & \multicolumn{2}{c}{ Phase Current } \\
Ripple & Uncoupled & Coupled & Uncoupled & Coupled \\
\hline Non-Interleaved & 1 & 1 & 1 & 1 \\
\hline Interleaved & $\Gamma$ & $\Gamma$ & 1 & $\frac{1+\beta \Gamma}{1+\beta}$ \\
\hline
\end{tabular}

of the multiple magnetic legs are added together with ripple reduced, which greatly reduces the magnetic flux ripple in the center leg of the coupled inductor, yielding greatly reduce phase current ripple. For weak coupling, i.e., $\beta \rightarrow+\infty$, the circuit reduces to $M$ parallel connected capacitor branches with a shared and shorted returning path. The voltage across the current source is determined by the voltage of the branch capacitor only, with no ripple cancellation across phases.

\section{B. Output Current Ripple Reduction}

Following the same superposition analysis, the output current ripple of an interleaved PWM coupled inductor is

$$
\Delta i_{\text {out }}^{\text {interleaved }}=M \Gamma \Delta v_{L x}^{*}+M \Delta v_{C}^{*}=M \sigma \Gamma\left(M \mathcal{R}_{C}+\mathcal{R}_{L}\right) .
$$

For an non-interleaved coupled inductor,

$$
\Delta i_{\text {out }}^{\text {non-interleaved }}=M \sigma\left(M \mathcal{R}_{C}+\mathcal{R}_{L}\right) .
$$

The output ripple reduction ratio for a PWM coupled inductor between interleaved and non-interleaved operation is

$$
\gamma_{\text {out }} \stackrel{\text { def }}{=} \frac{\Delta i_{\text {out }}^{\text {interleaved }}}{\Delta i_{\text {out }}^{\text {non-interleaved }}}=\Gamma
$$

As indicated, the impact of interleaving on output current ripple reduction in coupled inductors is the same as in noncoupled inductors. The coupling coefficient $\beta$ has no impact on the output ripple reduction.

\section{INTERLEAVING AND Multiphase COUPLing}

Interleaving and multiphase coupling are two effective and complementary way of creating mutual advantages in multicell PWM converters. Tables I compares the normalized output and phase current ripples for multiphase PWM converters with and without interleaving, and with and without coupling. In summary, interleaving can help to reduce the output current ripple regardless of the coupling coefficient across phases. The phase current ripple can be reduced by interleaving only if the multiple phases are strongly coupled.

\section{VERIFICATION AND EXTENDED Discussions}

Tabel II lists and compares the ripple reduction ratio derived in previous literature and in this work. The results shown in this letter is more concise and intuitive. We perform SPICE simulation on interleaved and non-interleaved, and coupled and uncoupled 4-phase buck converter with parameters listed in Fig. 7 to verify the effectiveness of the models. The waveforms in Fig. 8 and Fig. 9 show that: 
TABLE II

Phase CurRent Ripple Reduction Ratios

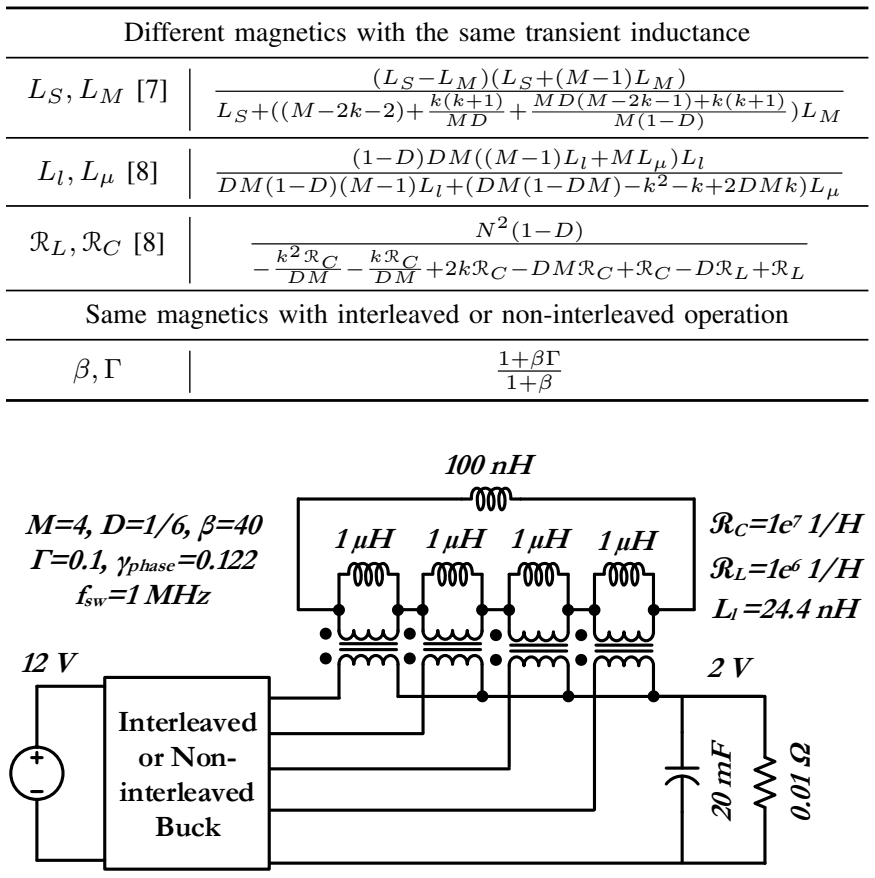

Fig. 7. An example 4-phase coupled inductor buck converter.

- The large-signal output dynamics of coupled, uncoupled, interleaved, non-interleaved conditions are the same. Interleaving and coupling only change the switching ripple. They don't change the large-signal output dynamics;

- Interleaving can reduce the output current ripple by a factor $\Gamma$ for both coupled and uncoupled scenarios. In this example, $\Gamma=0.1$;

- If the circuit is both coupled and interleaved, the ripple reduction mechanism of interleaving is extended from output current ripple to phase current ripple, with the ripple reduction factor equals $\gamma_{\text {phase }}$. In practise, $\gamma_{\text {phase }}$ is always larger than $\Gamma$ due to the unavoidable leakage flux. In this example, $\gamma_{\text {phase }}=0.122$.

\section{CONCLUSION}

This letter theoretically investigates the ripple reduction mechanism of pulsed-width-modulated (PWM) coupled inductors in interleaving operation by applying circuit duality and superposition. The analysis provides useful guidelines for designing interleaved and coupled PWM converters.

\section{REFERENCES}

[1] B. Miwa, "Interleaved Conversion Techniques for High Density Power Supplies," PhD Thesis, M.I.T., 1992.

[2] C. Chang and M. A. Knights, "Interleaving technique in distributed power conversion systems," IEEE Trans. on Circuits and Systems I: Fund. Theory and Applic., vol. 42, no. 5, May 1995.

[3] D. J. Perreault and J. G. Kassakian, "Distributed interleaving of paralleled power converters," IEEE Trans. on Circ. and Sys. I: Fund. Theo. and Applic., vol. 44, no. 8, pp. 728-734, Aug. 1997.

[4] D. Baba, "Benefits of a Multiphase Buck Converter," Texas Instruments Analog Applications Journal, Oct. 2012.
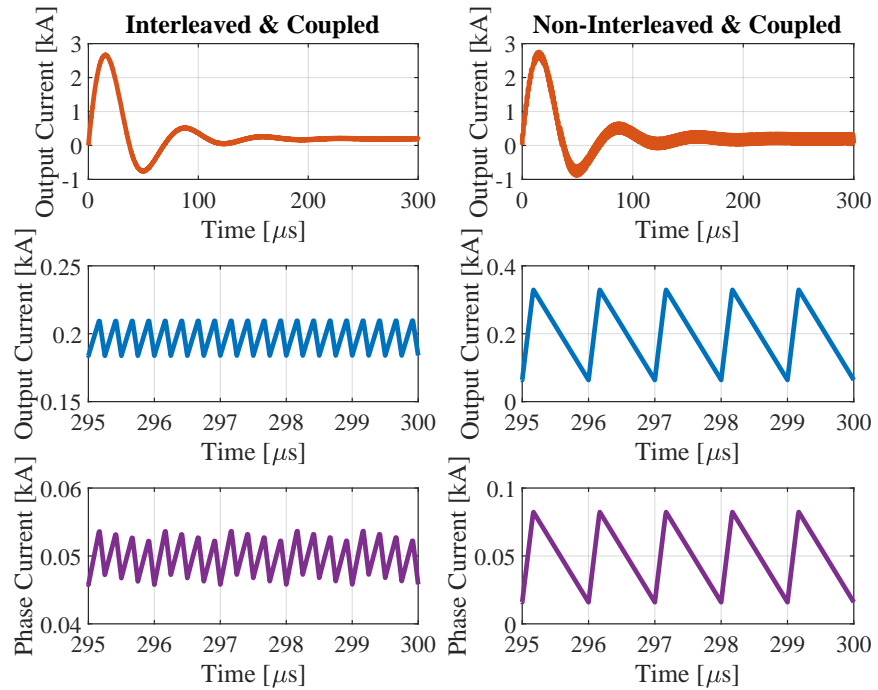

Fig. 8. Simulated output and phase current for the 4-phase coupled buck converter in Fig. 7 in interleaved and non-interleaved operation.
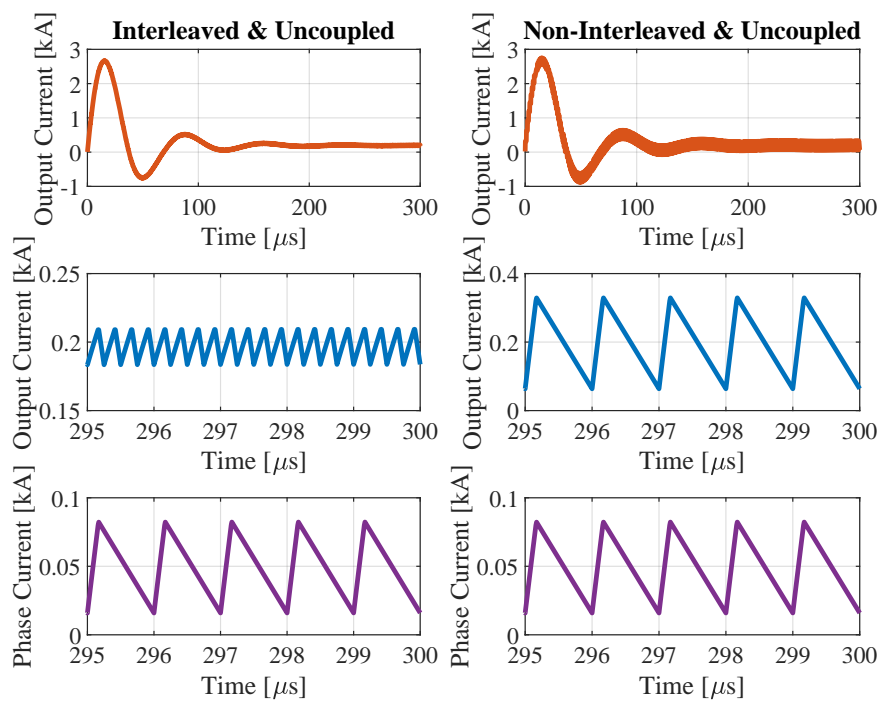

Fig. 9. Simulated output and phase current waveforms for a 4-phase uncoupled buck converter with the same leakage inductance as the one in Fig. 7, in interleaved and non-interleaved operation, respectively.

[5] C. R. Sullivan and M. Chen, "Coupled Inductors for FastResponse High-Density Power Delivery: Discrete and Integrated," IEEE Custom Integrated Circuits Conference (CICC), 2021.

[6] J. Li, C. R. Sullivan and A. Schultz, "Coupled inductor design optimization for fast-response low-voltage DC-DC converters," IEEE Applied Power Electron. Conference and Exposition, 2002.

[7] Y. Dong, "Investigation of multiphase coupled-inductor buck converters in point-of-load applications", PhD Thesis, Virginia Tech, 2009.

[8] M. Chen and C. R. Sullivan, "Unified Models for Coupled Inductors Applied to Multiphase PWM Converters," IEEE Transactions on Power Electronics, accepted.

[9] S. El-Hamamsy and E. I. Chang, "Magnetics modeling for computer-aided design of power electronics circuits," IEEE Power Electron. Special. Conf., Milwaukee, WI, USA, 1989.

[10] D. C. Hamill, "Lumped equivalent circuits of magnetic components: the gyrator-capacitor approach," IEEE Transactions on Power Electronics, vol. 8, no. 2, pp. 97-103, April 1993. 